\title{
Impact of Pulmonary Tuberculosis on the EGFR Mutational Status and Clinical Outcome in Patients with Lung Adenocarcinoma
}

\section{In Kyoung Hwang, MD \\ Seung Sook Paik, MD \\ Seung Hyeun Lee, MD, PhD²}

\begin{abstract}
Purpose
Although it has been suggested that pulmonary tuberculosis (TB) is associated with increased risk of lung cancer, the exact mechanism is not clearly identified. We investigated the effect of pulmonary TB on the epidermal growth factor receptor (EGFR) mutational status and clinical outcome in patients with pulmonary adenocarcinoma.
\end{abstract}

\section{Materials and Methods}

We reviewed data of patients diagnosed with pulmonary adenocarcinoma harboring EGFR mutations and treated at our institution from 2008 to 2015. We divided our population into two groups: patients with pre-existing TB lesions on chest computed tomography scan (TB group) and those without the lesions (non-TB group). We compared the differences in EGFR mutational status, response to tyrosine kinase inhibitors (TKIs) and survival between the two groups.

\section{Results}

A total of 477 patients with pulmonary adenocarcinoma were analyzed. One hundred eightythree patients (39\%) had EGFR-mutated tumors and 100 (21\%) patients had pre-existing TB lesions. The frequency of EGFR mutation was significantly higher in the TB group compared with the non-TB group ( $56 \%$ vs. $34 \%, p=0.038$ ). Pre-existing TB lesions were independently associated with more frequent EGFR mutations in multivariate analysis (odds ratio, 1.43). In addition, both the progression-free survival (9.1 months vs. 11.6 months, $p=0.020)$ and the overall survival (19.4 months vs. 24.5 months, $p=0.014)$ after first-line EGFR-TKIs were significantly shorter in the TB group than in the non-TB group.

\section{Conclusion}

Previous pulmonary TB may be associated with more frequent EGFR mutations and poorer treatment response to EGFR-TKIs in patients with pulmonary adenocarcinoma.
Medicine, Department of Internal Medicine,

Kyung Hee University School of Medicine,

23 Kyungheedae-ro, Dongdaemun-gu,

Seoul 02447, Korea

Tel: 82-2-958-8511

Fax: 82-2-968-1848

E-mail: humanmd04@hanmail.net

Received February 1, 2018

Accepted March 30, 2018

Published Online April 2, 2018

\author{
Key words \\ Lung neoplasms, Adenocarcinoma, Tuberculosis, \\ Epidermal growth factor receptor, Mutation, \\ Tyrosine kinase inhibitors, Response
}

\section{Introduction}

Lung cancer and pulmonary tuberculosis (TB) are two major public health issues associated with significant morbidity and mortality. Lung cancer is the leading cause of cancer-related deaths worldwide [1]. It accounts for approximately $23 \%$ of all cancer deaths and 88,655 patients died from lung cancer during period of 2008 to 2012 in Korea [2]. Lung cancer has been associated with a variety of benign lung conditions such as chronic obstructive pulmonary disease (COPD), idiopathic pulmonary fibrosis (IPF), and TB [3-5]. TB is one of the major causes of death among infectious diseases. In 2016, 10.4 million people fell ill with TB and 1.8 million died from the disease worldwide according to the World Health Organization [6].

Several prospective and retrospective studies have suggested that TB is associated with an increased risk of lung 
cancer [7-9]. Chronic inflammation leading to imbalance in DNA damage and repair mechanisms may be a possible pathophysiology of lung cancer in TB patients. A study showed that chronic airway inflammation promoted lung cancer development in a KRAS-mutated mouse model [10]. Moreover, chronic pulmonary TB infection resulted in dysplasia and squamous cell carcinoma of lung following accumulation of genomic alterations and dysregulation of growth factors [11].

Epidermal growth factor receptor (EGFR) gene encodes signaling proteins crucial for cell proliferation and survival, and EGFR mutations are major driver mutations occurring in lung adenocarcinomas [12]. Approximately 15\% of white patients and $30 \%-40 \%$ of Asian patients with lung adenocarcinoma carried tumors harboring EGFR mutations [13]. Deletion of exon 19 and L858R point mutations in exon 21 are the most common gene alterations in patients with lung adenocarcinoma. The tumors harboring these mutations showed dramatic response rates $(73 \%-91 \%)$ and prolonged progression-free survival (PFS) (7.7-13.3 months) when treated with EGFR tyrosine kinase inhibitors (TKIs) [14-16]. The EGFR mutations are more frequent in women, East-Asian and never smokers [17]. Interestingly, previous studies suggested the possible association between chronic inflammation and EGFR signaling. Casalino-Matsuda et al. [18] showed that oxidant-induced goblet cell metaplasia in human bronchial epithelial cells induces EGFR activation. In addition, TBinduced overexpression of epiregulin is associated with invasiveness of EGFR-mutated cell [19]. However, there is scarce data whether pulmonary TB was associated with increased frequency of driving mutations and its role in clinical outcome of patients with lung cancer.

As Korea is among countries with the highest prevalence of TB in general population and EGFR mutation frequency in lung cancer, it is plausible that frequent EGFR mutations may be attributed to high TB prevalence. Thus, we investigated whether previous pulmonary $\mathrm{TB}$ affects the EGFR mutational status or clinical outcomes of the patients with pulmonary adenocarcinoma.

\section{Materials and Methods}

\section{Patients and data collection}

We retrospectively screened patients with histologically or cytologically confirmed pulmonary adenocarcinoma who were diagnosed and treated at Dongnam Institute of Radiological \& Medical Sciences, a secondary referral hospital in Busan, South Korea, from January 2008 through December
2015. Patients with the results of EGFR mutation testing available were included in this study. We excluded patients who had active pulmonary TB along with lung cancer and who were diagnosed with other cancers within 5 years since lung cancer diagnosis, as their clinical course may be influenced by those comorbidities. We reviewed the electronic medical records to obtain demographic and clinical data including age, sex, Eastern Cooperative Oncology Group (ECOG) performance status, smoking status, TNM stage, systemic or pulmonary comorbidities and tumor mutational status. All patients underwent chest computed tomography, brain magnetic resonance imaging, and ${ }^{18} \mathrm{~F}$-fluorodeoxyglucose positron-emission tomography (PET) for clinical staging determined according to the International Association for the Study of Lung Cancer TNM staging classification of nonsmall cell lung cancer [13]. Tumor response was examined by computed tomography (CT) every two cycles of systemic treatment and evaluated according to the Response Evaluation Criteria in Solid Tumors (RECIST) ver. 1.1 [20]. In case of EGFR-TKIs, a single cycle comprised 4 weeks of treatment.

We reviewed initial chest CT scan at diagnosis to determine concomitant old TB lesions. We divided our lung cancer population into two groups: patients with old TB lesions (TB group) and those without lesions (non-TB group). Old TB lesions were defined as at least one of the following as previously described [21]: fibrotic scar with volume loss, fibronodular lesions, calcified or non-calcified granulomas, or pleural thickening. The existence and location of old TB lesions were determined based on the consensus of two independent reviewers (I.K. Hwang and S.S. Paik). In case of ambiguous findings, consensus was arrived at after discussion with a chest radiologist at our institution. In case of difficult diagnosis of old TB lesions and lung cancer-related or metastatic lesions based on chest $\mathrm{CT}$, the final decision was made by comparison of the corresponding images with those of PET. To exclude patients with active pulmonary TB, we confirmed the negative results of polymerase chain reaction for TB, acid-fast bacilli staining and TB culture using sputum or bronchial washing specimens obtained at the time of lung cancer diagnosis.

\section{EGFR mutation testing}

Genomic DNA was extracted from formalin-fixed, paraffin-embedded, 5 - $\mu \mathrm{m}$-thick tissue sections using the High Pure polymerase chain reaction (PCR) Template Preparation Kit (Roche Applied Science, Mannheim, Germany) according to the manufacturer's instructions. The extracted DNA was stored at $-20^{\circ} \mathrm{C}$ until analysis. EGFR Pyro Kit (QIAGEN Korea Ltd., Seoul, Korea) and PyroMark Q24 System (QIAGEN Korea Ltd.) were used to detect EGFR mutations by real-time PCR. The primer sets cover mutations or deletions 
Table 1. Characteristics of study population

\begin{tabular}{|c|c|c|c|c|}
\hline & \multirow{2}{*}{$\begin{array}{c}\text { No. of } \\
\text { patients }(\%)\end{array}$} & \multicolumn{2}{|c|}{ Groups } & \multirow{2}{*}{ p-value } \\
\hline & & TB group & Non-TB group & \\
\hline All & $477(100)$ & $100(21)$ & $377(79)$ & \\
\hline \multicolumn{5}{|l|}{ Sex } \\
\hline Female & $197(41)$ & $37(37)$ & $160(42)$ & 0.077 \\
\hline Male & $280(59)$ & $63(63)$ & $217(58)$ & \\
\hline \multicolumn{5}{|l|}{ Age (yr) } \\
\hline$<65$ & $304(64)$ & $43(43)$ & $172(46)$ & 0.531 \\
\hline$\geq 65$ & $173(36)$ & $57(57)$ & $205(54)$ & \\
\hline \multicolumn{5}{|l|}{ Smoking } \\
\hline Never & $205(43)$ & $33(33)$ & $172(46)$ & 0.061 \\
\hline Ever & $272(57)$ & $67(67)$ & $205(54)$ & \\
\hline \multicolumn{5}{|l|}{ ECOG performance status } \\
\hline 0,1 & $395(83)$ & $80(80)$ & $315(84)$ & 0.692 \\
\hline$\geq 2$ & $82(17)$ & $20(20)$ & $62(16)$ & \\
\hline \multicolumn{5}{|l|}{ Stage } \\
\hline I-IIIA & $225(47)$ & $60(60)$ & $165(44)$ & 0.084 \\
\hline IIIB-IV & $252(53)$ & $40(40)$ & $212(56)$ & \\
\hline \multicolumn{5}{|c|}{ Location of primary cancer mass } \\
\hline Central & $114(24)$ & $22(22)$ & $92(24)$ & 0.318 \\
\hline Peripheral & $362(76)$ & $78(78)$ & $284(76)$ & \\
\hline \multicolumn{5}{|c|}{ Location of old TB lesion and cancer } \\
\hline Ipsilateral another lobe & $38(38)$ & $38(38)$ & - & - \\
\hline Same lobe & $36(36)$ & $36(36)$ & - & \\
\hline Contralateral lung & $26(26)$ & $26(26)$ & - & \\
\hline \multicolumn{5}{|c|}{ Underlying systemic diseases } \\
\hline None & $207(44)$ & $43(43)$ & $164(44)$ & 0.457 \\
\hline HTN/IHD & $182(39)$ & $39(39)$ & $143(38)$ & \\
\hline $\mathrm{DM}$ & $27(6)$ & $6(6)$ & $21(6)$ & \\
\hline CKD & $15(3)$ & $5(5)$ & $10(3)$ & \\
\hline Others & $37(8)$ & $3(3)$ & $34(9)$ & \\
\hline \multicolumn{5}{|l|}{ Underlying lung diseases } \\
\hline None & $413(87)$ & $82(82)$ & $330(88)$ & 0.096 \\
\hline COPD & $42(9)$ & $11(11)$ & $31(8)$ & \\
\hline ILD & $15(3)$ & $4(4)$ & $8(2)$ & \\
\hline Bronchiectasis & $7(1)$ & $3(3)$ & $6(2)$ & \\
\hline \multicolumn{5}{|l|}{$K R A S^{\text {a) }}$} \\
\hline Wild-type & $177(89)$ & $43(91)$ & $132(88)$ & 0.341 \\
\hline Mutated & $22(11)$ & $4(9)$ & $18(12)$ & \\
\hline \multicolumn{5}{|l|}{$A L K$ translocation ${ }^{\text {a) }}$} \\
\hline Absent & $228(95)$ & $45(100)$ & $183(95)$ & 0.533 \\
\hline Present & $10(5)$ & 0 & $10(5)$ & \\
\hline \multicolumn{5}{|l|}{ EGFR } \\
\hline Wild-type & $294(61)$ & $44(44)$ & $250(66)$ & 0.038 \\
\hline Mutated & $183(39)$ & $56(56)$ & $127(34)$ & \\
\hline Exon19 deletion & $93(19)$ & $33(33)$ & $60(16)$ & \\
\hline L858R mutation & $78(16)$ & $20(20)$ & $58(14)$ & \\
\hline Other sites & $12(3)$ & $3(3)$ & $9(5)$ & \\
\hline
\end{tabular}

(Continued to the next page) 
Table 1. Continued

\begin{tabular}{|c|c|c|c|c|}
\hline & \multirow{2}{*}{$\begin{array}{c}\text { No. of } \\
\text { patients }(\%)\end{array}$} & \multicolumn{2}{|c|}{ Groups } & \multirow{2}{*}{ p-value } \\
\hline & & TB group & $\overline{\text { Non-TB group }}$ & \\
\hline \multicolumn{5}{|c|}{ EGFR-TKI use (any treatment lines) } \\
\hline No & 330 (69) & $68(68)$ & $262(69)$ & 0.956 \\
\hline Yes & $147(31)$ & $32(32)$ & $115(31)$ & \\
\hline
\end{tabular}

ECOG, Eastern Cooperative Oncology Group; HTN, hypertension; IHD, ischemic heart disease; DM, diabetes mellitus; CKD, chronic kidney disease; COPD, chronic obstructive pulmonary disease; ILD, interstitial lung disease, KRAS, Kirsten rat sarcoma; ALK, anaplastic lymphoma kinase; EGFR-TKI, epidermal growth factor receptor tyrosine kinase inhibitor. ${ }^{a} K R A S$ mutation and $A L K$ translocation data were available in 199 and 238 patients, respectively.

spanning exons 18 to 21 of the genes encoding tyrosine kinase domain of EGFR. Details of these methods were described in a previous study [22].

\section{Statistical analyses}

Statistical analyses were performed using the SPSS ver. 20.0 software for Windows (IBM Corp., Armonk, NY). PFS was defined as the time from the first day of chemotherapy to disease progression or death from any cause. Overall survival (OS) was defined from the start day of systemic treatment for advanced disease or the day of surgical resection for early-stage disease to death from any cause. Associations between categorical variables were compared using the chisquared test. Associations between clinicopathological parameters and survival were evaluated by univariate analysis using the log-rank test. Subsequently, multivariate Cox proportional hazards regression analysis was conducted after adjusting for parameters with p-values of $<0.2$ in the univariate analysis. Survival probability was estimated by the Kaplan-Meier method. A p-value of $<0.05$ was considered statistically significant.

\section{Ethical statement}

Informed consents were obtained from all patients alive and the study protocol was approved by the Institutional Review Board of the Dongnam Institute of Radiological \& Medical Sciences (D-1705-002-002).

\section{Results}

\section{Patient characteristics}

During the study period, 513 patients were diagnosed as lung adenocarcinoma at our institution. After exclusion of patients with cancers other than lung cancer $(n=11)$, active pulmonary TB at the initial diagnosis $(n=2)$ and without any available data for tumor EGFR mutational status $(n=23), 477$ patients were found eligible for this study. The clinicopathological characteristics of the patients are summarized in Table 1. All subjects were Korean with a median age of 64 years (range, 38 to 84 years). Old TB lesions on chest CT scan were detected in 100 patients (21\%, TB group) while no TB lesions in 377 patients (79\%, non-TB group). 280 patients $(58 \%)$ were male, 173 patients $(36 \%)$ were above the age of 65 years and 272 patients $(57 \%)$ were current or former smokers. 395 patients $(83 \%)$ had ECOG performance status of 0 or 1 , and 252 patients $(53 \%)$ were stage IIIB or IV. Tests for anaplastic lymphoma kinase $(A L K)$ translocation including immunohistochemistry and fluorescence in situ hybridization were performed in 238 patients (50\%), while KRAS mutation testing was performed in 199 patients (42\%); 10 patients $(4 \%)$ were positive for $A L K$ translocation and 22 patients $(11 \%)$ were positive for KRAS mutation. 270 patients $(57 \%)$ had more than one systemic comorbidity including hypertension and diabetes while 64 patients $(13 \%)$ had benign lung diseases other than TB. One hundred fortyseven patients $(30 \%)$ received EGFR-TKIs during their clinical course.

\section{Comparison of EGFR mutational status}

Overall, 183 patients (39\%) carried EGFR-mutated tumors. The most frequent mutation was deletion in exon $19(50 \%)$ followed by L858R point mutation (43\%). The frequency of 
Table 2. Analysis results for the factors affecting frequency of EGFR mutations

\begin{tabular}{|c|c|c|c|c|}
\hline & \multicolumn{2}{|c|}{ Univariate analysis } & \multicolumn{2}{|c|}{ Multivariate analysis } \\
\hline & OR $(95 \% \mathrm{CI})$ & p-value ${ }^{a)}$ & OR $(95 \% \mathrm{CI})$ & $\overline{p-v^{2}\left(u e^{b)}\right.}$ \\
\hline \multicolumn{5}{|l|}{ Sex } \\
\hline Female & $2.65(1.08-1.93)$ & $<0.001$ & $1.21(1.05-1.86)$ & 0.034 \\
\hline Male & Reference & & Reference & \\
\hline \multicolumn{5}{|l|}{ Age (yr) } \\
\hline$<65$ & $2.14(1.57-2.76)$ & 0.163 & $1.08(0.64-2.13)$ & 0.521 \\
\hline$\geq 65$ & Reference & & Reference & \\
\hline \multicolumn{5}{|l|}{ Smoking } \\
\hline Never & $1.82(1.13-2.17)$ & $<0.001$ & $1.27(1.12-1.98)$ & 0.044 \\
\hline Ever & Reference & & Reference & \\
\hline \multicolumn{5}{|c|}{ Old TB lesions } \\
\hline No & Reference & 0.014 & Reference & 0.031 \\
\hline Yes & $1.79(1.15-2.24)$ & & $1.43(1.08-2.47)$ & \\
\hline
\end{tabular}

EGFR, epidermal growth factor receptor; $\mathrm{OR}$, odds ratio; $\mathrm{CI}$, confidence interval; $\mathrm{TB}$, tuberculosis. ${ }^{\mathrm{a}}$ Univariate analysis p-value by log-rank test, ${ }^{b}$ Multivariate analysis p-value by Cox regression.

Table 3. Frequency of EGFR mutations according to the spatial relationship between the primary lung mass and old TB lesions in the TB group

\begin{tabular}{|c|c|c|c|c|c|c|c|c|}
\hline & \multicolumn{4}{|c|}{ EGFR mutation } & \multicolumn{4}{|c|}{ Exon 19 mutation } \\
\hline & $\begin{array}{c}\text { All } \\
(n=100)\end{array}$ & $\begin{array}{c}\text { Positive } \\
(n=56)\end{array}$ & $\begin{array}{c}\text { Negative } \\
(\mathrm{n}=44)\end{array}$ & p-value & $\underset{(n=77)}{\text { All }}$ & $\begin{array}{c}\text { Positive } \\
(n=33)\end{array}$ & $\begin{array}{l}\text { Negative } \\
(n=44)\end{array}$ & p-value \\
\hline \multicolumn{9}{|l|}{$\begin{array}{l}\text { Location of lung cancer and } \\
\text { TB lesions }\end{array}$} \\
\hline Same lobe & 38 & $23(61)$ & $15(39)$ & 0.028 & 30 & $17(57)$ & $13(43)$ & 0.013 \\
\hline Ipsilateral different lobe & 36 & $26(72)$ & $10(28)$ & & 27 & $14(52)$ & $13(48)$ & \\
\hline Contralateral lung & 26 & $7(27)$ & $19(73)$ & & 20 & $2(10)$ & $18(90)$ & \\
\hline
\end{tabular}

Values are presented as number (\%). EGFR, epidermal growth factor receptor; TB, tuberculosis.

EGFR mutations was significantly higher in the TB group than in the non-TB group $(56 / 100,56 \%$ vs. $127 / 377,34 \%$; $\mathrm{p}=0.038$ ). In addition, the frequency of exon 19 deletion in the TB group was significantly higher than in the non-TB group $(33 / 100,33 \%$ vs. $60 / 377,16 \%$; $p=0.011)$, while the frequency of L858R point mutation was not different between the two groups $(20 / 100,20 \%$ vs. $58 / 377,15 \%$; $\mathrm{p}=0.254)$ (Table 1).

\section{Multivariate analysis for the prediction of EGFR muta- tion}

To determine the factors associated with the rate of EGFR mutation, we performed a multivariate analysis for possible clinical variables. As presented in Table 2, after adjusting for possible confounders, female sex (odds ratio [OR], 1.21; 95\% confidence interval [CI], 1.05 to 1.86), never smoking (OR, 1.27; 95\% CI, 1.12 to 1.98), and old TB lesions (OR, 1.43; $95 \%$ CI, 1.08 to 2.47 ) were independently associated with an increased rate of $E G F R$ mutation.

\section{Association between EGFR mutation and the location of old TB lesion}

To investigate the relationship between EGFR mutations and location of old TB lesions, we analyzed the frequency of EGFR mutations according to the spatial relationship between the primary lung mass and old TB lesions. As summarized in Table 3, among 100 patients in the TB group, 38 $(38 \%)$ and 36 patients $(36 \%)$ had the main tumor mass in the 
Table 4. Overall survival analyses results according to clinicopathological parameters of all study subjects

\begin{tabular}{|c|c|c|c|c|c|}
\hline & \multirow{2}{*}{$\begin{array}{l}\text { Median overall } \\
\text { survival (mo) }\end{array}$} & \multicolumn{2}{|c|}{ Univariate analysis } & \multicolumn{2}{|c|}{ Multivariate analysis } \\
\hline & & HR $(95 \%$ CI) & p-value $e^{a}$ & HR (95\% CI) & p-value ${ }^{b)}$ \\
\hline All & 40.5 & & & & \\
\hline \multicolumn{6}{|l|}{ Sex } \\
\hline Female & 49.3 & Reference & 0.007 & Reference & 0.204 \\
\hline Male & 28.9 & $1.47(1.05-3.19)$ & & $1.19(0.74-3.72)$ & \\
\hline \multicolumn{6}{|l|}{ Age (yr) } \\
\hline$<65$ & 53.4 & Reference & 0.003 & Reference & 0.158 \\
\hline$\geq 65$ & 29.1 & $1.55(1.16-2.07)$ & & $1.26(0.91-1.75)$ & \\
\hline \multicolumn{6}{|l|}{ Smoking } \\
\hline Never & 46.7 & Reference & 0.031 & Reference & 0.910 \\
\hline Ever & 27.8 & $1.38(1.04-1.85)$ & & $1.18(0.60-1.61)$ & \\
\hline \multicolumn{6}{|c|}{ ECOG performance status } \\
\hline 0,1 & 53.3 & Reference & $<0.001$ & Reference & 0.008 \\
\hline$\geq 2$ & 13.1 & $3.75(2.72-5.16)$ & & $1.67(1.14-2.43)$ & \\
\hline \multicolumn{6}{|l|}{ Stage } \\
\hline I-IIIA & 101.4 & Reference & $<0.001$ & Reference & $<0.001$ \\
\hline IIIB-IV & 18.3 & $5.79(4.06-8.28)$ & & $3.61(2.31-5.64)$ & \\
\hline \multicolumn{6}{|c|}{ Underlying systemic diseases } \\
\hline None & 43.3 & Reference & 0.112 & Reference & 0.457 \\
\hline Yes & 26.7 & $1.61(0.89-2.90)$ & & $1.14(0.45-2.47)$ & \\
\hline \multicolumn{6}{|c|}{ Underlying lung diseases other than ТB } \\
\hline None & 44.3 & Reference & 0.226 & NA & \\
\hline Yes & 24.8 & $1.75(0.98-2.11)$ & & & \\
\hline \multicolumn{6}{|l|}{$K R A S^{c)}$} \\
\hline Wild-type & 40.5 & Reference & 0.759 & NA & \\
\hline Mutated & 31.0 & $1.09(0.57-2.10)$ & & & \\
\hline \multicolumn{6}{|c|}{$A L K$ translocation ${ }^{\mathrm{c}}$} \\
\hline Absent & 46.0 & Reference & 0.737 & NA & \\
\hline Present & 28.8 & $1.42(0.26-2.62)$ & & & \\
\hline \multicolumn{6}{|l|}{ EGFR } \\
\hline Wild-type & 24.1 & $1.97(1.04-3.65)$ & $<0.001$ & $1.91(1.29-3.58)$ & $<0.001$ \\
\hline Mutated & 59.0 & Reference & & Reference & \\
\hline \multicolumn{6}{|c|}{ EGFR-TKI use (any treatment lines) } \\
\hline No & 46.4 & Reference & 0.699 & NA & \\
\hline Yes & 37.5 & $1.06(0.79-1.43)$ & & & \\
\hline \multicolumn{6}{|c|}{ Old TB lesions } \\
\hline No & 45.2 & Reference & 0.154 & Reference & 0.273 \\
\hline Yes & 38.1 & $1.32(0.59-1.77)$ & & $1.18(0.47-2.65)$ & \\
\hline
\end{tabular}

HR, hazard ratio; CI, confidence interval; ECOG, Eastern Cooperative Oncology Group; TB, tuberculosis; KRAS, Kirsten rat sarcoma; ALK, anaplastic lymphoma kinase; EGFR-TKI, epidermal growth factor receptor tyrosine kinase inhibitor; NA, not analyzed. ${ }^{\mathrm{a})}$ Univariate analysis $\mathrm{p}$-value by log-rank test, ${ }^{\mathrm{b}}$ Multivariate analysis $\mathrm{p}$-value by Cox regression, ${ }^{\mathrm{c}} \mathrm{KRAS}$ mutation and $A L K$ translocation data were available in 199 and 238 patients, respectively.

same lobe and different lobe of the ipsilateral lung containing the main TB lesion, respectively, while 26 patients $(26 \%)$ had main tumor mass in the contralateral lung. The frequency of EGFR mutation in patients who had the main mass in the same lobe and different lobe of the ipsilateral lung with the main TB lesion were $61 \%$ (23 among 38 patients) and $72 \%$ (26 among 36 patients), respectively, while that of those who had lung cancer in the contralateral lung is $27 \%$ (7 among 26 patients). The difference in EGFR mutational frequencies according to the location of lung cancer and TB lesions was 
Table 5. Survival analyses results according to clinicopathologic parameters in patients treated with first-line EGFR-TKIs

\begin{tabular}{|c|c|c|c|c|c|c|c|}
\hline & \multirow[b]{2}{*}{$\begin{array}{c}\text { No. of } \\
\text { patients }(\%)\end{array}$} & \multicolumn{3}{|c|}{ PFS } & \multicolumn{3}{|c|}{ Os } \\
\hline & & $\begin{array}{l}\text { Median } \\
\text { PFS (mo) }\end{array}$ & $\begin{array}{c}\text { Univariate } \\
\text { analysis } \\
\text { p-value }\end{array}$ & $\begin{array}{c}\text { Multivariate } \\
\text { analysis adjusted } \\
\text { HR ( } 95 \% \text { CI) }\end{array}$ & $\begin{array}{l}\text { Median } \\
\text { OS (mo) }\end{array}$ & $\begin{array}{c}\text { Univariate } \\
\text { analysis } \\
\text { p-value }\end{array}$ & $\begin{array}{c}\text { Multivariate } \\
\text { analysis adjusted } \\
\text { HR (95\% CI) }\end{array}$ \\
\hline All & $88(100)$ & 10.5 & & & 21.5 & & \\
\hline \multicolumn{8}{|l|}{ Sex } \\
\hline Female & $23(26)$ & 11.1 & 0.254 & NA & 22.8 & 0.215 & NA \\
\hline Male & $65(74)$ & 9.4 & & & 21.1 & & \\
\hline \multicolumn{8}{|l|}{ Age (yr) } \\
\hline$<65$ & $31(35)$ & 10.6 & 0.606 & NA & 23.6 & 0.099 & Reference \\
\hline$\geq 65$ & $57(65)$ & 9.5 & & & 20.3 & & $1.18(0.73-1.93)$ \\
\hline \multicolumn{8}{|l|}{ Smoking } \\
\hline Never & $25(28)$ & 9.8 & 0.311 & NA & 24.6 & 0.003 & Reference \\
\hline Ever & $63(72)$ & 10.3 & & & 19.0 & & $2.02(1.47-3.84)$ \\
\hline \multicolumn{8}{|l|}{ ECOG PS } \\
\hline 0,1 & $59(67)$ & 11.8 & 0.048 & Reference & 23.7 & 0.203 & NA \\
\hline$\geq 2$ & $29(33)$ & 9.6 & & $1.27(0.79-2.05)$ & 21.2 & & \\
\hline \multicolumn{8}{|l|}{ Stage } \\
\hline IIIB & $25(28)$ & 11.9 & 0.019 & Reference & 24.8 & 0.011 & Reference \\
\hline IV & $63(72)$ & 8.9 & & $1.76(1.10-2.83)$ & 18.1 & & $1.84(1.09-3.10)$ \\
\hline \multicolumn{8}{|c|}{ Old TB lesions } \\
\hline No & $67(76)$ & 11.6 & 0.020 & Reference & 24.5 & 0.014 & Reference \\
\hline Yes & $21(24)$ & 9.1 & & $1.85(1.16-3.56)$ & 19.4 & & $1.60(1.16-3.74)$ \\
\hline
\end{tabular}

EGFR-TKI, epidermal growth factor receptor tyrosine kinase inhibitor; PFS, progression-free survival; OS, overall survival; HR, hazard ratio; CI, confidence interval; NA, not analyzed; ECOG, Eastern Cooperative Oncology Group; PS, performance status; TB, tuberculosis.

statistically significant $(\mathrm{p}=0.028)$. In case of exon 19 deletion, the frequency in patients who had the main mass in the same lobe and different lobe of the ipsilateral lung with the main TB lesion were 57\% (17 among 30 patients) and 52\% (14 among 27 patients), respectively, whereas that of those who had lung cancer in the contralateral lung is 10\% (2 among 20 patients). The difference in the frequencies of exon 19 deletion according to the location of lung cancer and TB lesions was also statistically significant $(\mathrm{p}=0.013)$.

\section{Survival analyses for overall population and sub-popu- lation according to EGFR mutational status}

To evaluate whether patients with TB-related lung adenocarcinoma showed different survival rates compared with those without TB lesions, we performed a survival analysis according to clinicopathological parameters of all study subjects. The results of survival analyses are summarized in Table 4 . The median OS in all study subjects was 40.5 months (range, 3.3 to 65.7 months). Univariate analysis revealed that male gender, old age, ever smoking, poor performance sta- tus, advanced stage and EGFR wild-type were significantly associated with shorter OS. The presence of old TB lesions was not associated with $\mathrm{OS}(\mathrm{p}=0.154)$. Multivariate analysis showed that poor performance status (hazard ratio [HR], $1.67 ; 95 \% \mathrm{CI}, 1.14$ to 2.43$)$, advanced stage (HR, 3.61; $95 \% \mathrm{CI}$, 2.31 to 5.64 ) and EGFR wild-type (HR, 1.91; 95\% CI, 1.29 to 3.58) were independently associated with shorter OS. We further performed subgroup analyses according to EGFR mutational status. However, OS was similar between the two groups regardless of EGFR mutational status.

\section{Survival analysis of patients receiving EGFR-TKIs as a first-line treatment}

To investigate whether the pulmonary $\mathrm{TB}$ affects the response to TKI treatment, we compared the PFS and OS in patients who were treated with EGFR-TKIs as a first-line treatment in each group. Among all the patients, 88 (18.5\%) were treated with EGFR-TKIs such as gefitinib $(n=39)$, erlotinib ( $\mathrm{n}=28)$, and afatinib $(\mathrm{n}=21)$ for locally advanced or metastatic EGFR-mutated lung adenocarcinoma. Twenty-one 
A

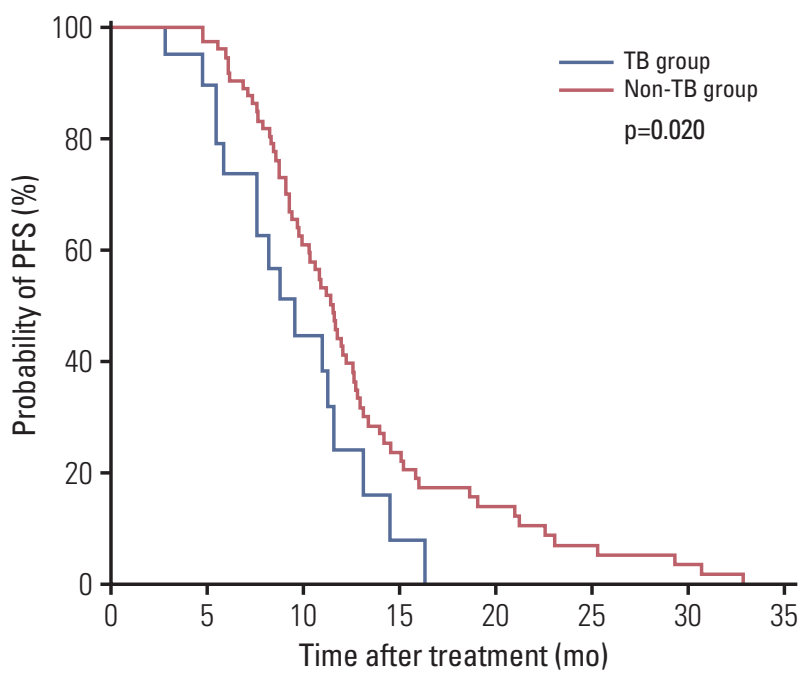

Fig. 1. Kaplan-Meier survival curves for progression-free survival (A) and overall survival (B) in patients treated with epidermal growth factor receptor (EGFR) tyrosine kinase inhibitors for EGFR-mutated lung adenocarcinoma. p-values were determined using the log-rank test. PFS, progression-free survival; OS, overall survival; TB, tuberculosis.

and 67 patients were included in TB group and non-TB group, respectively. As first-line EGFR-TKI was approved by Korea Food \& Drug Administration at April 2010, the enrolment period for this subgroup was 2010-2015. First-line gefitinib and erlotinib were covered by National Health Insurance System (NHIS) of Korea since April 2011 and March 2013, respectively. Ten patients (4 for gefitinib, 6 for erlotinib) paid for their own first-line EGFR-TKI treatment before the reimbursement of each TKI. First-line afatinib was available since October 2014 and the proportion of patients who were treated with this drug as a first-line therapy was similar in both groups $(23 \%$ [5/21] in TB group and 24\% [16/67] in non-TB group). The number of patients who received firstline EGFR-TKIs and EGFR-TKIs used was distributed throughout the study period, and they are summarized in S1 Table. The survival analyses results are summarized in Table 5. The median PFS of the 88 patients treated with EGFR-TKIs were 10.5 months (range, 4.6 to 21.2 months). In the univariate analysis, poor ECOG performance and advanced stage were significantly associated with shorter PFS. In addition, pulmonary TB lesions was significantly associated with shorter PFS (9.1 months vs. 11.6 months, $\mathrm{p}=0.020$ ). Multivariate analysis showed that advanced stage (HR, 1.76; 95\% CI, 1.10 to 2.83 ) and old TB lesions (HR, 1.85; 95\% CI, 1.16 to 3.56 ) were independently associated with shorter PFS. The Kaplan-Meier survival curves also showed that patients with old TB lesions were likely to have shorter PFS (Fig. 1A). The median OS of the 88 patients were 21.5 months (range, 5.1 to

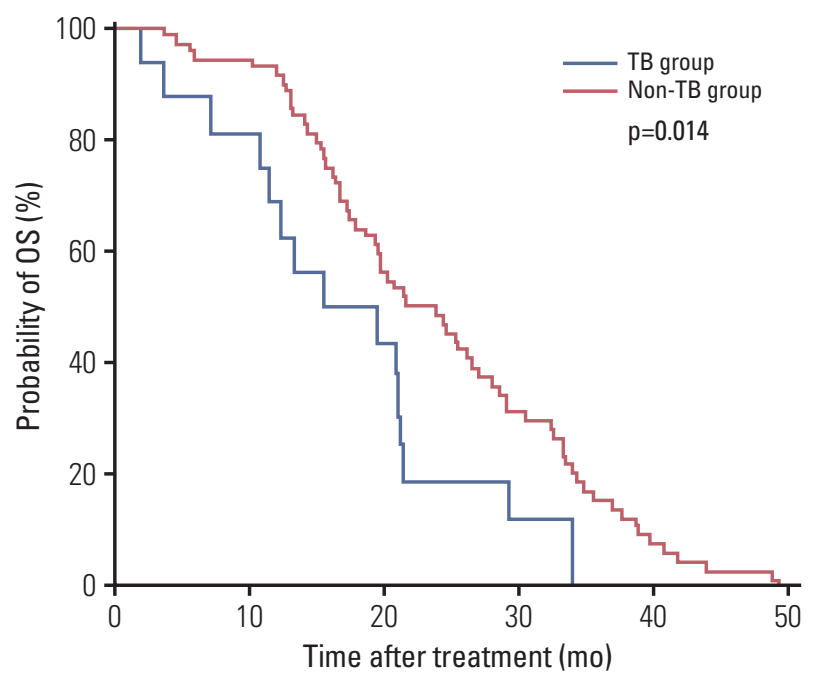

39.6 months). In the univariate analysis, ever smoking and advanced stage were associated with shorter OS. In addition, TB lesions was also associated with shorter OS (19.4 months vs. 24.5 months, $\mathrm{p}=0.014)$. Multivariate analysis showed that ever smoking ( $\mathrm{HR}, 2.02 ; 95 \% \mathrm{CI}, 1.47$ to 3.84), advanced stage (HR, 1.84; 95\% CI, 1.09 to 3.10) and old TB lesions (HR, 1.60; $95 \% \mathrm{CI}, 1.16$ to 3.74$)$ were independently associated with shorter OS. The Kaplan-Meier survival curves also showed that patients with old TB lesions were likely to have shorter OS (Fig. 1B).

\section{Discussion}

The current data demonstrate that pre-existing TB lesions are significantly associated with increased rate of EGFR mutation, especially exon 19 deletions in patients with lung adenocarcinoma. The mutation rate is significantly higher in patients carrying the main mass at the same lobe or in a different lobe of the ipsilateral lung with the main TB lesion compared with those who had main mass in the contralateral lung. In addition, patients with TB-related adenocarcinoma showed a poor treatment response and survival compared with non-TB-related counterparts when treated with EGFRTKIs. To the best of our knowledge, this is the second study demonstrating the association between pulmonary TB and 
the EGFR mutational status and the first study identifying the clinical impact of pulmonary TB on the clinical outcome of patients treated with first-line EGFR-TKIs for EGFRmutated lung adenocarcinoma.

It has been known that benign lung diseases such as COPD, IPF and TB are independently associated with the risk of lung cancer [23]. The mechanisms underlying the role of those diseases on lung cancer risk are still unclear; however, chronic inflammation seems to be a major contributor. A meta-analysis of 37 case-control and four cohort studies showed 1.8-fold increased risk of lung cancer among patients with pulmonary ТВ [24]. In that study, the association was significant only with adenocarcinoma, while no significant associations with squamous or small cell type of lung cancer were observed [24]. In a recent large-scale population-based cohort study using patient care data of Taiwan, the incidence of lung cancer was approximately 11 -fold higher in the population with a history of TB compared with those without past TB (26.3 vs. 2.4 per 10,000 person-years) [25]. The HR was 4.37 (95\% CI, 3.56 to 5.36) for the TB cohort after adjusting for demographic variables [25].

In the present study, $21 \%$ of all the study population carried old TB lesions revealed by chest $\mathrm{CT}$. This proportion was much higher than expected before the study. Previous studies reported that $2.1 \%$ to $13.1 \%$ of lung cancer patients had pre-existing ТВ [24-26]. However, the prevalence of preexisting TB in those studies may be underestimated as they collected TB information indirectly using International Classification of Diseases (ICD) codes from the nationwide database or a relatively small set of autopsy cases rather than a comprehensive review of chest images of lung cancer patients as reported in our present study. In a study of Taiwanese lung cancer cohort in which patients' chest CT was reviewed, the prevalence of old TB lesions in lung cancer patients was approximately $26 \%$ [27], which was comparable to our current data. As Taiwan and Korea are endemic areas for $\mathrm{TB}$, the high rates of old $\mathrm{TB}$ lesions among the lung cancer patients were attributed to the high prevalence of TB in both countries. Further large-scale investigation is warranted to confirm whether this rate reflected the prevalence in the general population or merely indicated regional trends.

Although TB-related chronic inflammation is believed to be a main mechanism of lung carcinogenesis, whether previous pulmonary $\mathrm{TB}$ is associated with increased rate of driving mutations of lung cancer is largely unknown. As Korea is an endemic area of TB with an incidence of 100 per 100,000 individuals [28] and known for high EGFR mutation rate in lung cancer [13], we had postulated that previous pulmonary TB infection may affect to the increased EGFR mutation rate in Korean population. Our data showed that EGFR mutation was more frequent in patients with old TB lesions than those without. Moreover, EGFR mutations were detected at a higher frequency in patients carrying the main cancer mass in the same lobe or in a different lobe of the ipsilateral lung containing the main TB lesions compared with those who did not. Those results are consistent with those of a previous study demonstrating a high occurrence of lung cancer in the lung ipsilateral to the old TB lesion and the risk of ipsilateral lung cancer remained elevated throughout the follow-up period [29]. Although further studies are required, those previous findings and ours suggest that TB-associated chronic and local inflammation may be involved in lung carcinogenesis by provoking genetic alterations including EGFR mutations.

To date, only a single study evaluated the association between previous pulmonary TB and frequency of EGFR mutation in lung cancer. Luo et al. [27] evaluated the association in 275 Taiwanese patients whose EGFR mutation results were available. In their study, overall 191 patients $(69.5 \%)$ had EGFR mutations and the EGFR mutation rate was significantly higher in patients with old TB lesions compared to those without the lesions ( $80.6 \%$ vs. $65.5 \%, \mathrm{p}=0.018$ ). In addition, they reported a high rate of exon 19 deletions in patients with old TB lesions that those without (68\% vs. 34\%, $\mathrm{p}<0.001$ ). Although the higher rate of EGFR mutation in patients with old TB lesions is in line with our study, the mutational frequency of the total population $(69.5 \%)$ was much higher than that of our population (39\%) and that of a previously report on Taiwanese population [13]. We enrolled much more patients with available EGFR mutation results $(\mathrm{n}=477)$ and the overall frequency of EGFR mutations was similar to that of a previous study [13]. In terms of survival, Luo et al. [27] reported poorer 1-year survival rate in patients with old TB lesions than those without $(77 \%$ vs. $85 \%$, $\mathrm{p}=0.015$ ). In addition, the poor survival rate of patients with TB lesions was consistent regardless of EGFR mutational status. These are different from our findings demonstrating no OS differences between the two groups. This discrepancy can be explained by different characteristics of study populations in the two studies. In Luo et al.'s study [27], most patients had locally advanced or metastatic cancers (83\%) and many of them were treated with EGFR-TKIs (79.3\%). However, our patients were evenly distributed in terms of stage, and thus $53 \%$ of them had advanced diseases and 30\% were treated with EGFR-TKIs during their clinical course. The differential survival between the two groups in the previous study by Luo et al. can be attributed to TKIs treatment in advanced diseases rather than the true effect of pulmonary TB on the prognosis of lung cancers of different stages. Whether previous pulmonary TB is a prognostic factor in lung cancer warrants further investigations.

We demonstrated poor clinical outcome in patients with old TB lesions receiving EGFR-TKIs as a first-line treatment compared to those without TB lesions. In a retrospective 
cohort study by Chang et al. [30] reviewing 8,265 patients from National Health Insurance Research Database of Taiwan, pulmonary TB history was associated with poor clinical response to EGFR-TKIs in male patients but with a better response in female patients. However, this study has limitations associated with heterogeneous patient data as all patients who received TKIs were enrolled regardless of EGFR mutational status or lines of treatment. In our study, we analyzed the PFS and OS of patients who were treated with first-line EGFR-TKIs for EGFR-mutated lung adenocarcinomas. Our results showing poorer PFS and OS in patients with old TB lesions treated with EGFR-TKIs are partly consistent with aforementioned data. Although evaluation of genderbased impact was not possible because of the small population in our treatment group, the previous and our data suggest that pulmonary TB may adversely affect the TKI response in patients with EGFR-mutated pulmonary adenocarcinoma. The reason for the poor response to EGFR-TKIs in TB-related lung cancer is not clear; however, aggressive phenotype induced by chronic inflammation may be a possible explanation. Zhang et al. [19] demonstrated that chronic TB infection upregulated epiregulin, which correlated with the invasiveness of EGFR-mutant lung cancer. Further investigations are required to elucidate the mechanism of adverse effect of previous TB to TKI treatment.

There are several limitations in our study. First, this is a retrospective study in which selection bias is inevitable. Second, cumulative incidence of lung cancer in TB patients or dominant histologic types in TB-related lung cancer could not be evaluated in our population. Third, we provided only limited information on other genetic alterations including $A L K$ rearrangement or KRAS mutation. Comprehensive genomic analysis using next-generation sequencing of large cohorts may elucidate the clinical impact of pulmonary TB on the development of genetic alterations during lung carcinogenesis. To confirm our findings and evaluate the association between previous pulmonary TB and genetic alternations of lung cancer, we have initiated data collection from the National Health Insurance Service Database of our country.

In summary, the current data suggest that pulmonary TB influences not only the EGFR mutational status but also treatment response of the patients treated with EGFR-TKIs. Our findings highlight the clinical implications of previous pulmonary TB during lung carcinogenesis and on the clinical outcome after lung cancer treatment, and warrant future investigations for the mechanism of poor response to TKIs and the relevance with a variety of genetic alterations in TB-related lung adenocarcinoma.

\section{Electronic Supplementary Material}

Supplementary materials are available at Cancer Research and Treatment website (https: // www.e-crt.org).

\section{Conflicts of Interest}

Conflict of interest relevant to this article was not reported.

\section{Acknowledgments}

This work was supported by the National Research Foundation of Korea (NRF) grant funded by the Ministry of Science, ICT \& Future Planning (grant No. NRF-2017R1C1B5016828). We also appreciate Dr. Ji-yeon Han of the Department of Radiology, Dongnam Institute of Radiological \& Medical Sciences for her enthusiastic comments and advice.

\section{References}

1. Bray F, Jemal A, Grey N, Ferlay J, Forman D. Global cancer transitions according to the Human Development Index (20082030): a population-based study. Lancet Oncol. 2012;13:790801.

2. Park JY, Jang SH. Epidemiology of lung cancer in Korea: recent trends. Tuberc Respir Dis. 2016;79:58-69.

3. Brenner DR, Boffetta P, Duell EJ, Bickeboller H, Rosenberger A, McCormack V, et al. Previous lung diseases and lung cancer risk: a pooled analysis from the International Lung Cancer Consortium. Am J Epidemiol. 2012;176:573-85.

4. Hubbard R, Venn A, Lewis S, Britton J. Lung cancer and cryptogenic fibrosing alveolitis: a population-based cohort study. Am J Respir Crit Care Med. 2000;161:5-8.
5. Durham AL, Adcock IM. The relationship between COPD and lung cancer. Lung Cancer. 2015;90:121-7.

6. World Health Organization. Global tuberculosis report 2017 [Internet]. Geneva: World Health Organization; 2017 [cited 2017 Nov 11]. Available from: http://www.who.int/tb/publications/global_report/en.

7. Hong S, Mok Y, Jeon C, Jee SH, Samet JM. Tuberculosis, smoking and risk for lung cancer incidence and mortality. Int J Cancer. 2016;139:2447-55.

8. Wu CY, Hu HY, Pu CY, Huang N, Shen HC, Li CP, et al. Pulmonary tuberculosis increases the risk of lung cancer: a population-based cohort study. Cancer. 2011;117:618-24.

9. Everatt R, Kuzmickiene I, Davidaviciene E, Cicenas S. Inci- 
dence of lung cancer among patients with tuberculosis: a nationwide cohort study in Lithuania. Int J Tuberc Lung Dis. 2016;20:757-63.

10. Moghaddam SJ, Li H, Cho SN, Dishop MK, Wistuba, II, Ji L, et al. Promotion of lung carcinogenesis by chronic obstructive pulmonary disease-like airway inflammation in a K-rasinduced mouse model. Am J Respir Cell Mol Biol. 2009;40:44353.

11. Nalbandian A, Yan BS, Pichugin A, Bronson RT, Kramnik I. Lung carcinogenesis induced by chronic tuberculosis infection: the experimental model and genetic control. Oncogene. 2009;28:1928-38.

12. Jett JR, Carr LL. Targeted therapy for non-small cell lung cancer. Am J Respir Crit Care Med. 2013;188:907-12.

13. Shi Y, Au JS, Thongprasert S, Srinivasan S, Tsai CM, Khoa MT, et al. A prospective, molecular epidemiology study of EGFR mutations in Asian patients with advanced non-small-cell lung cancer of adenocarcinoma histology (PIONEER). J Thorac Oncol. 2014;9:154-62.

14. Mok TS, Wu YL, Thongprasert S, Yang CH, Chu DT, Saijo N, et al. Gefitinib or carboplatin-paclitaxel in pulmonary adenocarcinoma. N Engl J Med. 2009;361:947-57.

15. Rosell R, Carcereny E, Gervais R, Vergnenegre A, Massuti B, Felip E, et al. Erlotinib versus standard chemotherapy as firstline treatment for European patients with advanced EGFR mutation-positive non-small-cell lung cancer (EURTAC): a multicentre, open-label, randomised phase 3 trial. Lancet Oncol. 2012;13:239-46.

16. Sequist LV, Yang JC, Yamamoto N, O'Byrne K, Hirsh V, Mok $\mathrm{T}$, et al. Phase III study of afatinib or cisplatin plus pemetrexed in patients with metastatic lung adenocarcinoma with EGFR mutations. J Clin Oncol. 2013;31:3327-34.

17. Chang YS, Choi CM, Lee JC. Mechanisms of epidermal growth factor receptor tyrosine kinase inhibitor resistance and strategies to overcome resistance in lung adenocarcinoma. Tuberc Respir Dis. 2016;79:248-56.

18. Casalino-Matsuda SM, Monzon ME, Forteza RM. Epidermal growth factor receptor activation by epidermal growth factor mediates oxidant-induced goblet cell metaplasia in human airway epithelium. Am J Respir Cell Mol Biol. 2006;34:581-91.

19. Zhang J, Iwanaga K, Choi KC, Wislez M, Raso MG, Wei W, et al. Intratumoral epiregulin is a marker of advanced disease in non-small cell lung cancer patients and confers invasive properties on EGFR-mutant cells. Cancer Prev Res (Phila). 2008;1: 201-7.

20. Hwang KE, Kim HR. Response evaluation of chemotherapy for lung cancer. Tuberc Respir Dis. 2017;80:136-42.

21. Kim YI, Goo JM, Kim HY, Song JW, Im JG. Coexisting bronchogenic carcinoma and pulmonary tuberculosis in the same lobe: radiologic findings and clinical significance. Korean J Radiol. 2001;2:138-44.

22. Kim TJ, Park CK, Yeo CD, Park K, Rhee CK, Kim J, et al. Simultaneous diagnostic platform of genotyping EGFR, KRAS, and ALK in 510 Korean patients with non-small-cell lung cancer highlights significantly higher ALK rearrangement rate in advanced stage. J Surg Oncol. 2014;110:245-51.

23. Denholm R, Schuz J, Straif K, Stucker I, Jockel KH, Brenner $\mathrm{DR}$, et al. Is previous respiratory disease a risk factor for lung cancer? Am J Respir Crit Care Med. 2014;190:549-59.

24. Liang HY, Li XL, Yu XS, Guan P, Yin ZH, He QC, et al. Facts and fiction of the relationship between preexisting tuberculosis and lung cancer risk: a systematic review. Int J Cancer. 2009;125:2936-44.

25. Yu YH, Liao CC, Hsu WH, Chen HJ, Liao WC, Muo CH, et al. Increased lung cancer risk among patients with pulmonary tuberculosis: a population cohort study. J Thorac Oncol. 2011;6:32-7.

26. Cicenas S, Vencevicius V. Lung cancer in patients with tuberculosis. World J Surg Oncol. 2007;5:22.

27. Luo YH, Wu CH, Wu WS, Huang CY, Su WJ, Tsai CM, et al. Association between tumor epidermal growth factor receptor mutation and pulmonary tuberculosis in patients with adenocarcinoma of the lungs. J Thorac Oncol. 2012;7:299-305.

28. Lee SH. Tuberculosis infection and latent tuberculosis. Tuberc Respir Dis. 2016;79:201-6.

29. Yu YY, Pinsky PF, Caporaso NE, Chatterjee N, Baumgarten $\mathrm{M}$, Langenberg $\mathrm{P}$, et al. Lung cancer risk following detection of pulmonary scarring by chest radiography in the prostate, lung, colorectal, and ovarian cancer screening trial. Arch Intern Med. 2008;168:2326-32.

30. Chang CH, Lee CH, Ho CC, Wang JY, Yu CJ. Gender-based impact of epidermal growth factor receptor mutation in patients with nonsmall cell lung cancer and previous tuberculosis. Medicine (Baltimore). 2015;94:e444. 